\title{
Wide Bezel Televisions Decrease Immersive Experiences
}

\author{
Hwayeon Kong $(\mathbb{D}$ \\ Department, Human ICT Convergence, Sungkyunkwan University, Seoul, Republic of Korea \\ Correspondence should be addressed to Hwayeon Kong; flohello@gmail.com
}

Received 19 February 2020; Revised 7 August 2020; Accepted 11 August 2020; Published 1 September 2020

Academic Editor: Alice Chirico

Copyright (c) 2020 Hwayeon Kong. This is an open access article distributed under the Creative Commons Attribution License, which permits unrestricted use, distribution, and reproduction in any medium, provided the original work is properly cited.

This study explored how telepresence could be affected by stimuli from reality that distracts people while they are watching television. The sample comprised of 36 undergraduate and graduate students from a university in South Korea (age range: 18-38 years, $M=22.61$, and $\mathrm{SD}=4.12$ ). A between-subjects experimental design was employed with two types of viewing equipment (a television screen vs. a television screen with side screens that act as stimuli from reality) and two bezel widths $(2 \mathrm{~cm} v s .10 \mathrm{~cm})$ to examine how each condition influenced the viewers' perceived telepresence. The results revealed that participants' perception of telepresence was not affected by the type of viewing equipment. However, the level of telepresence was affected by the bezel width: the thinner the bezel, the more telepresence felt by the viewers. These findings provide important insights that can guide the future designs of screen bezels for televisions and other devices in order to more effectively create immersive virtual worlds. Future studies are needed to examine the relationship between central vision and telepresence.

\section{Introduction}

At the 2018 Consumer Electronics Show (CES), it was predicted that televisions (TVs) would remain as a core home appliance within the near future because of their higher picture quality via larger screens. Ever since their development in the 19th century, TVs have continually evolved, first to color TVs, then to digital TVs, and now to internet-enabled TVs that can utilize various applications.

TVs are anticipated to remain the most popular home appliance for entertainment media [1-4]. As a result, many studies have been conducted on user experience (UX) to improve the satisfaction of TV users [5-8], and one critical factor involved is "telepresence" (TP). Skarbez and colleagues [9] cited Gibson [10] in their definition of the term "telepresence": "Telepresence can be thought of as the experience of one's physical environment; it refers not to one's surroundings as they exist in the physical world, but to the perception of those surroundings as mediated by both automatic and controlled mental process [10]: telepresence is defined as the sense of being in an environment."

TP has been variously defined and given meanings based on the particular situation of diverse media $[11,12]$. It is explained as the immersion of users [13] in the experiential environment created through various media [14]. TP also occurs while watching TV and is a crucial component of UX that allows viewers to enjoy and consume TV content [15-18]. TP can be estimated by an index of the degree of viewers' immersion in the videos or content that they enjoy and understand [19-21].

In the same context, TP is defined as a feeling of being in another environment (i.e., virtual environments such as those of games, movies, and TV programs) created via information and communication technology (ICT) [22-25].

In an experiment, Vettehen et al. [19] investigated the effect of a 360-degree format for conveying news stories. They conducted an experiment to explore more persuasive news video formats (360-degree video vs. traditional 2D video) and mainly used the concept of telepresence to evaluate the effect of the media. It was found that telepresence mediated how enjoyable and credible the viewers found the media to be.

Tijus et al. [20] evaluated the effects of high-definition television (HDTV) on presence and found that HDTV increased the images' legibility and amount of "reading." They interpreted that this was due to telepresence-that participants with a higher feeling of telepresence had a higher comprehension of what was being shown, stimulating viewers' curiosity and imagination. 
Additionally, another study [21] investigated telepresence to explore in greater detail the difference between the conditions of viewing sports content at a movie theatre and at home. The researchers focused on the telepresence when watching mediated sports and identified that it would determine the suspenseful nature of the media experience as well as the subsequent enjoyment.

In this study, we refer to the feeling of being in the virtual environment as TP and the feeling of being in the physical environment as real presence (RP). Viewers experience TP through visual and sound stimulation from TV screens and speakers. Each of the sensory stimuli is received by the viewer's sensory-motor system, where the TV stimuli override the sensory stimuli from reality and impact the viewer's sensory system. This in turn provides the viewer with the feeling of $\mathrm{TP}$ and causes the viewer to regard the virtual environment as the existing real-world space. Thus, we may consider that TP and RP are negatively correlated, wherein the enhancement of TP weakens RP and vice versa.

So far, research on TV UX has mainly focused on factors that directly increase the level of TP, with barely [26-29] any focus on factors that indirectly increase the level of TP by decreasing the level of RP [30-32]. Therefore, this study investigates RP factors and the relationship of RP and TP within the TV UX.

There has been research on how components such as screen size, resolution, and curvature are related to UX [26-29]. However, there is still a lack of studies on bezel-a space or frame around a display device-which is also an aspect of screens. Therefore, we will explore how TP is affected by bezels, which are both TV form factors.

Thus, we arrived at two research questions:

(1) May stimuli outside the virtual environment decrease the level of TP?

(2) May TV bezel's width decrease the level of RP and indirectly increase the level of TP?

Researchers have reported that in the perceptual system of TV viewers, the perceptual stimulation of the virtual environment generated by TV competes with the perceptual stimulation of the real-world environment in which the viewer exists [30-32]. As a result, it has been argued that the feeling of existing in a location is mostly determined by which environment (physical environment vs. virtual environment) provides relatively more stimuli information. This is defined as sensory saturation or "the percentage of the sensory channel occupied by stimuli (information) from the virtual as opposed to the physical environment" [30].

Through the concept "breaks in presence," Slater and Steed [31, 33-35] also explained how TP can be converted into RP and weaken TP. In general, TV viewers receive signals (sensory stimuli) from both the virtual and real worlds. However, only the stimuli from one of the worlds can be dominant in determining the feeling of presence, based on consistent cognitive Gestalt [31, 33]. Further, it is said that [31] "sensory data corresponding to the nonfavored interpretation may be ignored or incorporated into the prevailing Gestalt” [33]. This means that an individual has the ability to pay selective attention to and respond to a set of stimuli from one environment and ignore all stimuli from other environments [36].

Sheridan [32] argued that factors unrelated to video content can also weaken $\mathrm{TP}$, such as a blocked view or external noise while watching TV, process interruptions (e.g., messages such as notifications and announcements, malfunctions, and error messages), awkward camera walking, slow update speeds, the sound of a phone ringing, and broadcast station logos on the edge of the screen.

In addition to the aforementioned points, TV specifications such as screen length, width, and bezel are factors that can be visually perceived while viewing TVs. A bezel is a component of TVs belonging to reality, so the bezel's physical presence is expected to play a role in decreasing the viewer's level of TP. Since a bezel's physical presence is an interrupting factor, RP is expected to increase with an increase in the width of the bezel, while TP is expected to change inversely.

Based on the literature and our research questions, we framed two hypotheses to be tested.

(i) Hypothesis 1. Process interruptions by cues from the physical environment will negatively affect the subject's feeling of telepresence more than the absence of such cues from the real environment.

(ii) Hypothesis 2. A wider bezel will negatively affect the subject's feeling of telepresence more than a thinner bezel.

\section{Materials and Methods}

In our study, we manipulate stimuli outside of TP to test our first hypothesis. Two additional monitors were aligned on both sides of the main monitor and continually displayed images (e.g., pictures of scenery, buildings, and animals) in 5 -second and 10-second intervals that were not related to the story of the animation playing on the TV. The effective field of view (FOV) in humans covers roughly $200^{\circ}$ degrees horizontally [37]. So, the stimuli from these two peripheral monitors will draw the attention of the eye to the visual events [38], negatively influencing to create TP of the main monitor $[39,40]$.

2.1. Participants and Procedures. We had announced the study inviting students to participate in it on a South Korean university website. A total of 36 undergraduate and graduate students were voluntarily recruited in this manner.

The experiment used a 2 (side screen interruptions: yes vs. no) $\times 2$ (bezel width: $10 \mathrm{~cm}$ vs. $2 \mathrm{~cm}$ ) balanced, betweensubjects design.

Data gathering took place at a laboratory at the university. On the day of the experiment, the researchers explained the experimental procedure and the required time commitment. Participants were assured prior to the experiment that any information provided by them in the survey would be kept confidential. They were also asked if they felt any discomfort participating in the experiment. 
After each participant gave his/her consent to participate, they were instructed to sit on a chair $150 \mathrm{~cm}$ away from a table with a TV $(58 \mathrm{~cm} \times 36 \mathrm{~cm}$ screen $)$ to watch a 7 -minute short animated film (The Passenger; http://www.chrisj.com. au). Following this, participants participated in a questionnaire survey through a desktop which was outside of the laboratory. After the experiment, participants were given KRW 5,000 as an incentive.

All procedures in this study were performed in accordance with the ethical standards of the institutional and/or national research committee and with the 1964 Helsinki Declaration and its later amendments or comparable ethical standards.

2.2. Manipulation of Real-World Stimuli. In this study, we defined "the virtual world" as the world created through TV and "the real world" as the world in which the viewer actually exists. To test our first hypothesis, we considered visual stimulation through the monitor to be stimuli from reality. That is, when the subject experiences the virtual world through the main monitor, the visual stimuli are made using real-world stimuli through the side monitors. These side monitors were aligned on both sides of the main monitor and continually displayed images (e.g., pictures of scenery, buildings, and animals) in 5-second and 10-second intervals that were not related to the story of the animation playing on the TV.

To test the second hypothesis, we strengthened the stimulus by thickening the bezel width. The bezel thickness of the monitor used in the study was $2 \mathrm{~cm}$, and the bezel's visual stimuli was produced by applying a black frame to the bezel to produce a monitor with a $10 \mathrm{~cm}$ bezel.

In order to test the impact of the increased bezel width, we presented real-sized photos of a $2 \mathrm{~cm}$ bezel screen and a $10 \mathrm{~cm}$ bezel screen at the same time to the participants and asked them to find the differences; all 20 participants responded that the $10 \mathrm{~cm}$ bezel was thicker than the $2 \mathrm{~cm}$ bezel.

2.3. Measures. Telepresence is a basic state of consciousness and fundamental property of consciousness [41]. It is an indicator itself or has a mediated effect for other perceptions and behaviors $[27,42]$. Because of this, we solely measured this particular indicator.

Kim and Biocca [43] suggested that telepresence was composed of two factors, which they labeled "arrival" (the feeling of being in another environment) and "departure" (the feeling of leaving the physical environment). This concept applies to the competition for our feeling of presence between a virtual environment and a physical environment. The present study used the telepresence scale developed by Kim and Biocca [43] with a few variations and each were measured $(M=46.94, \mathrm{SD}=12.36$, and Cronbach's alpha $=0.83$ ) on a 10-point Likert scale: (1) when the broadcast ended, I felt like I came back to the "real world" after a journey (from strongly disagree to strongly agree); (2) the television created a new world for me, and the world suddenly disappeared when the broadcast ended (from strongly disagree to strongly agree); (3) during the broadcast, I felt like I was inside the world that the television had created (from never to always); (4) during the broadcast, I never forgot the fact that I was in the middle of an experiment (from never to always; reversed scale); (5) during the broadcast, my body was inside the room, but my mind was inside the world created by the television (from never to always); (6) during the broadcast, the television-generated world was more real or present for me, as compared to the "real world" (from never to always); (7) the televisiongenerated world appeared to me as only "something I saw," rather than "somewhere I visited" (from never to always; reversed scale); (8) during the broadcast, my mind was inside the room, but not inside the world created by the television (from never to always; reversed scale).

\section{Results}

A two-way ANOVA was conducted, with the main screen on which participants were watching an animation (with vs. without side screens playing real-world stimuli) and the bezel width $(2 \mathrm{~cm}$ vs. $10 \mathrm{~cm})$ as independent variables.

There were no significant effects on the dependent variable of TP by the screen type (no interruption: $M=6.26$ and $\mathrm{SD}=1.43$; interruption by second screens: $M=5.37$ and $\mathrm{SD}=1.57)$. Therefore, hypothesis 1 -the existence of interruption by cues from the physical environment will negatively affect the subject's feeling of telepresence more than the absence of cues from the physical environment-is rejected.

However, the type of bezel presence- $2 \mathrm{~cm}$ vs. $10 \mathrm{~cm}$-had a significant main effect. The participants watching the animated movie on the TV with $2 \mathrm{~cm}$ bezels showed higher TP levels $(M=6.38$ and $\mathrm{SD}=1.16)$ than did those who watched the animated movie on the TV with $10 \mathrm{~cm}$ bezels $(M=5.40$ and $\mathrm{SD}=1.71), \quad F(1,36)=3.05$, $p<0.05$. Therefore, hypothesis 2 -the wider bezel will more negatively affect the subject's feeling of telepresence than the thinner bezel-is supported.

\section{Discussion}

We examined the relationship between television TP and distraction from the real environment. However, we found that the stimuli from the side screens did not critically influence the subject's sense of TP. This finding contradicts previously reported results that stimuli from the physical environment reduce TP during the experience of virtual reality $[30-32,39]$. It can be presumed that the interruptions suggested in this experiment may not have worked as expected. In other words, the real-world stimuli manipulated by the researchers may not have been visually perceived by the subjects while watching the video.

In human vision, the field of view (FOV) covers approximately $200^{\circ}$ horizontally [37]. The diameter of the field of view, however, varies upon the status of the viewer [38]. When a viewer is reading a book, driving a car, or focusing on a particular object, the FOV becomes the central vision (foveal vision) and only a narrow space within five degrees to 
the left and right dominates the visual perception [38]. It is possible that the real-world stimuli from the side screens (left-right) did not fall within the viewers' central vision and therefore did not play any role as visual stimuli to interrupt the TP of the participants.

In the second experiment, it was found that the level of bezel thickness as an RP factor negatively affects TP when watching TV. Analysis of the cases with the bezel of $10 \mathrm{~cm}$ width revealed that participants felt a lower level of TP, and analysis of the cases with the $2 \mathrm{~cm}$ bezel revealed that participants experienced a higher level of TP. These findings support prior studies that proposed that TP and RP have a competitive relationship that affects each other inversely as one is weakened or strengthened [30-32]. These results can be considered as the result of TP influenced by RP. Therefore, the physical presence of monitor bezels was confirmed as an important factor that can affect TP while watching TV.

However, there are some possible limitations in this experiment.

First, the sample size was not enough to represent the population of TV viewers-we confirmed that the results for the sample size in the experiment effect sizes [44] showed small-level effects $(d=0.14)$ among the four levels of effect: negligible (0.00-0.09), small (0.10-0.24), medium (0.25-0.39), and large (0.40+).

Another limitation that should be noted is that personal factors of the subjects were not considered in this study. Since the immersive tendency to experience presence is a personal factor related to cognitive competence, it makes theoretical sense that personality variables influence the tendency toward such a state, as well as the motivation to focus upon or attend to the media or task at hand. Viewers can respond differently to the same video images; some viewers are immersed while others are not, hence the importance of considering personal factors (e.g., personality or gender-related differences in the experience of presence) $[14,45,46]$. Researchers would greatly benefit from including these factors in future studies.

\section{Conclusions}

Despite these limitations, this study is significant in several ways. First, this study confirmed the importance of "central vision" [38] in TP via TV. We found that stimuli outside of the central vision area would not affect the viewers while they are immersed in screen content, but most studies have rarely addressed or considered the importance of the central vision [47-49].

We also found that the bezel negatively affected TP via TV. There have been attempts [50] to improve the UX concerning bezels, but the present study took a step further to confirm that the presence of bezels is a physical-environment stimulus with negative effects on TP. The findings provide important insights that will guide the future design of bezels for TVs to enhance viewers' TP.

\section{Data Availability}

The spreadsheet data used to support the findings of this study have been deposited in the Google Docs repository https://
docs.google.com/spreadsheets/d/1RybN7zH3be7BiWor2DW 9RbwX7nJPZISEDolnUjEAbmI/edit?usp=sharing.

\section{Conflicts of Interest}

The author declares that there are no conflicts of interest regarding the publication of this article.

\section{References}

[1] R. Lawler, "The TVs that mattered at CES," 2018, https://www. engadget.com/amp/2018/01/13/the-tvs-that-mattered-at-ces/.

[2] G. Von Krogh and E. Von Hippel, "The promise of research on open source software," Management Science, vol. 52, no. 7, pp. 975-983, 2006.

[3] J. Chang and J. Hwang, "The role of media in user participation: focusing on the knowledge activity in online space," Telematics and Informatics, vol. 51, p. 101407, 2020.

[4] W. Guebli and A. Belkhir, "TV home-box based IoT for smart home," in Proceedings of the Mediterranean Symposium on Smart City Application, pp. 1-7, Tangier, Morocco, October 2017.

[5] P. Desment and P. Hekkert, "Framework of product experience," International Journal of Design, vol. 1, no. 1, pp. 57-66, 2007.

[6] E. L.-C. Law, V. Roto, M. Hassenzahl, A. P. O. S. Vermeeren, and J. Kort, "Understanding, scoping and defining user experience," in Proceedings of the 27th International Conference On Human Factors in Computing Systems-CHI 09, pp. 719728, Boston, MA, USA, April 2009.

[7] K. A. Jeong, J. Han, N. Na, and H.-J. Suk, "Do consumers prefer curved monitors? Assessment of preferred curvature and readability performance," Journal of Information Display, vol. 18, no. 2, pp. 67-72, 2017.

[8] S. Park, J. Yi, D. Choi et al., "66-4: effects of display curvature and lateral viewing position on spatial presence and image quality for 55" TVs," SID Symposium Digest of Technical Papers, vol. 47, no. 1, pp. 911-914, 2016.

[9] R. Skarbez, F. P. Brooks Jr., and M. C. Whitton, "A survey of presence and related concepts," ACM Computing Surveys (CSUR), vol. 50, no. 6, p. 96, 2018.

[10] J. J. Gibson, The Ecological Approach to Visual Perception, Houghton Mifflin, Boston, MA, USA, 1979.

[11] W. Barfield, D. Zeltzer, T. Sheridan, and M. Slater, "Presence and performance within virtual environments," Virtual Environments and Advanced Interface Design, vol. 1, no. 1, pp. 473-513, 1995.

[12] M. Lombard, R. D. Reich, M. E. Grabe, C. C. Bracken, and T. B. Ditton, "Presence and television. The role of screen size," Human Communication Research, vol. 26, no. 1, pp. 75-98, 2000.

[13] B. G. Witmer and M. J. Singer, "Measuring presence in virtual environments: a presence questionnaire," Presence: Teleoperators and Virtual Environments, vol. 7, no. 3, pp. 225-240, 1998.

[14] J. Steuer, "Defining virtual reality: dimensions determining telepresence," Communication in the Age of Virtual Reality, pp. 33-56, Lawrence Erlbaum Associates, Hillsdale, NJ, USA, 1995.

[15] A. Bilgihan, F. Okumus, K. Nusair, and M. Bujisic, "Online experiences: flow theory, measuring online customer experience in e-commerce and managerial implications for the lodging industry," Information Technology \& Tourism, vol. 14, no. 1, pp. 49-71, 2014.

[16] V. Cauberghe, M. Geuens, and P. De Pelsmacker, "Context effects of TV programme-induced interactivity and telepresence on advertising responses," International Journal of Advertising, vol. 30, no. 4, pp. 641-663, 2011. 
[17] A. Mollen and H. Wilson, "Engagement, telepresence and interactivity in online consumer experience: reconciling scholastic and managerial perspectives," Journal of Business Research, vol. 63, no. 9-10, pp. 919-925, 2010.

[18] H. Tikkanen, J. Hietanen, T. Henttonen, and J. Rokka, "Exploring virtual worlds: success factors in virtual world marketing," Management Decision, vol. 47, no. 8, pp. 1357-1381, 2009.

[19] P. H. Vettehen, D. Wiltink, M. Huiskamp, G. Schaap, and P. Ketelaar, "Taking the full view: how viewers respond to 360degree video news," Computers in Human Behavior, vol. 91, pp. 24-32, 2019.

[20] C. Tijus, F. Levillain, H. Bessaa et al., "Immersive, emotive and cognitive effects of HDTV: evaluating effects of image definition on presence," in 2009 IEEE-RIVF International Conference On Computing and Communication Technologies, pp. 1-4, IEEE, Da Nang, Vietnam, July, 2009.

[21] K. Kim, Y. Cheong, and H. Kim, "The influences of sports viewing conditions on enjoyment from watching televised sports: an analysis of the FIFA World Cup audiences in theater vs. home," Journal of Broadcasting \& Electronic Media, vol. 60, no. 3, pp. 389-409, 2016.

[22] C. Heeter, "Being there: the subjective experience of presence," Presence: Teleoperators and Virtual Environments, vol. 1, no. 2, pp. 262-271, 1992.

[23] M. Slater and S. Wilbur, "A framework for immersive virtual environments (FIVE): speculations on the role of presence in virtual environments," Presence: Teleoperators and Virtual Environments, vol. 6, no. 6, pp. 603-616, 1997.

[24] C. C. Bracken, "Presence and image quality: the case of highdefinition television," Media Psychology, vol. 7, no. 2, pp. 191-205, 2005.

[25] M. Hartmann, "Numbers in the eye of the beholder: what do eye movements reveal about numerical cognition?" Cognitive Processing, vol. 16, no. 1, pp. 245-248, 2015.

[26] M. Söderlund, J. Colliander, and S. Szugalski, "Screen size effects in online data collections," Journal of Consumer Marketing, vol. 36, no. 6, pp. 751-759, 2019.

[27] H. Van Kerrebroeck, M. Brengman, and K. Willems, "When brands come to life: experimental research on the vividness effect of virtual reality in transformational marketing communications," Virtual Reality, vol. 21, no. 4, pp. 177-191, 2017.

[28] J. Brade, M. Lorenz, M. Busch, N. Hammer, M. Tscheligi, and P. Klimant, "Being there again-presence in real and virtual environments and its relation to usability and user experience using a mobile navigation task," International Journal of Human-Computer Studies, vol. 101, pp. 76-87, 2017.

[29] C. Peukert, J. Pfeiffer, M. Meißner, T. Pfeiffer, and C. Weinhardt, "Shopping in virtual reality stores: the influence of immersion on system adoption," Journal of Management Information Systems, vol. 36, no. 3, pp. 755-788, 2019.

[30] F. Biocca, "The cyborg's dilemma: progressive embodiment in virtual environments," Journal of Computer-Mediated Communication, vol. 3, no. 2, 1997.

[31] M. Slater and A. Steed, "A virtual presence counter," Presence: Teleoperators and Virtual Environments, vol. 9, no. 5, pp. 413-434, 2000.

[32] T. B. Sheridan, "Further musings on the psychophysics of presence," Presence: Teleoperators and Virtual Environments, vol. 5, no. 2, pp. 241-246, 1996.

[33] M. Slater, A. Brogni, and A. Steed, "Physiological responses to breaks in presence: a pilot study," in Proceedings of the 6th Annual International Workshop on Presence, Citeseer, San Diego, CA, USA, October 2003.
[34] M. Garau, D. Friedman, H. R. Widenfeld, A. Antley, A. Brogni, and M. Slater, "Temporal and spatial variations in presence: qualitative analysis of interviews from an experiment on breaks in presence," Presence: Teleoperators and Virtual Environments, vol. 17, no. 3, pp. 293-309, 2008.

[35] D. Slater, "Making things real," Theory, Culture \& Society, vol. 19, no. 5-6, pp. 227-245, 2002.

[36] K. E. Bystrom, W. Barfield, and C. Hendrix, "A conceptual model of the sense of presence in virtual environments," Presence: Teleoperators \& Virtual Environments, vol. 8, no. 2, pp. 24-244, 1999.

[37] J. W. Lin, H. B. L. Duh, D. E. Parker, H. Abi-Rached, and T. A. Furness, "Effects of field of view on presence, enjoyment, memory, and simulator sickness in a virtual environment," in Proceedings IEEE Virtual Reality 2002, pp. 164-171, IEEE, Orlando, FL, USA, March 2002.

[38] H. Gross, F. Blechinger, and B. Achtner, Handbook of Optical Systems, Survey of Optical Instruments, Wiley, Hoboken, NJ, USA, 2008.

[39] P. B. Seel, "Telepresence and immersion with ultra-highdefinition digital displays," in Digital Technology and the Future of Broadcasting: Global Perspectives, J. V. Pavlick, Ed., pp. 94-106, Routledge, London, UK, 2015.

[40] H. M. Assenheim, "Peripheral vision displays," Electro-Optical Displays, p. 311, Taylor and Francis, London, UK, 1992.

[41] J. M. Loomis, "Distal attribution and presence," Presence: Teleoperators and Virtual Environments, vol. 1, no. 1, pp. 113-119, 1992.

[42] S. J. Ahn and J. N. Bailenson, "Self-endorsing versus otherendorsing in virtual environments," Journal of Advertising, vol. 40, no. 2, pp. 93-106, 2011.

[43] T. Kim and F. Biocca, "Telepresence via television: two dimensions of telepresence may have different connections to memory and persuasion," Journal of Computer-Mediated Communication, vol. 3, no. 2, 2006.

[44] J. Cohen, Statistical Power Analysis for the Behavioral Sciences, Academic Press, Hillsdale, MI, USA, 2nd edition, 1988.

[45] S. Lee and G. J. Kim, "Effects of visual cues and sustained attention on spatial presence in virtual environments based on spatial and object distinction," Interacting with Computers, vol. 20, no. 4-5, pp. 491-502, 2008.

[46] J. Freeman, S. E. Avons, R. Meddis, D. E. Pearson, and W. IJsselsteijn, "Using behavioral realism to estimate presence: a study of the utility of postural responses to motion stimuli," Presence: Teleoperators and Virtual Environments, vol. 9, no. 2, pp. 149-164, 2000.

[47] P. B. Seel, "Background and future directions for research,"“Background and future directions for research," in Digital Technology and the Future of Broadcasting: Global Perspectives, J. V. Pavlick, Ed., p. 94, Routledge, Abingdon, UK, 2015.

[48] B. I. Olsen, B. Laeng, K.-A. Kristiansen, and G. Hartvigsen, "Size does matter: women mentally rotate large objects faster than men," Scandinavian Journal of Psychology, vol. 54, no. 3 , pp. 196-204, 2013.

[49] J. Ichino, K. Isoda, A. Hanai, and T. Ueda, "Effects of the display angle in museums on user's cognition, behavior, and subjective responses," in Proceedings of the SIGCHI Conference On Human Factors in Computing Systems, pp. 29792988, Paris, France, April 2013.

[50] D. E. Novy, "Computational immersive displays," Doctoral dissertation, Massachusetts Institute of Technology, Cambridge, MA, USA, 2013. 